Metab $\square \mathbf{C} \square \mathbf{u} \square \operatorname{erab} \square \mathbf{t} \square \square \square \square \mathbf{S} \square \square$

$\mathrm{s} \square \mathrm{aa} \square \square \mathrm{e} \square \square \mathrm{a}$

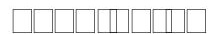

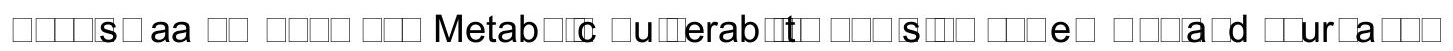

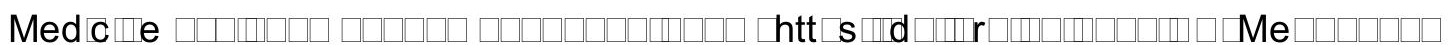

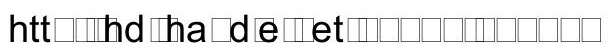

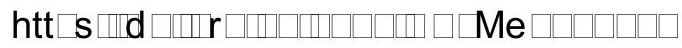

$\mathrm{u} \llbracket \mathrm{s} \square$ ec $\llbracket$ ed

$\ulcorner$ ub $\square$ shed $\square$ ers $\square \square$ 
be less suitable for routine treatment. Also, some patients may benefit more from firm adherence to high-potency dual antiplatelet therapy with either prasugrel or ticagrelor. In the COMPLETE trial, one quarter of the patients received clopidogrel, which may not be the most effective therapy in patients with acute coronary syndromes. ${ }^{10}$

Should the consistent lack of benefit with respect to all-cause mortality discourage the strategy of routine complete revascularization? Since this strategy appears to be safe and reduces the risk of the composite outcome of cardiovascular death or recurrent myocardial infarction, as well as the risk of future revascularization, it appears to be appropriate to recommend complete revascularization for patients similar to those included in the COMPLETE trial. We hope that the investigators will be able to obtain data from longer follow-up in order to evaluate whether the tendency toward a small reduction in all-cause mortality becomes significant over time. Better selection of high-risk patients may also refine the determination of who is most likely to benefit from complete revascularization. Regardless, in light of the results of the well-planned and well-executed trial by Mehta et al., the guidelines should recommend a strategy of full revascularization in patients with STEMI and multivessel disease, at least in those who have suitable nonculprit lesions.

Disclosure forms provided by the authors are available with the full text of this editorial at NEJM.org.

From the Department of Cardiology, Rigshospitalet, University of Copenhagen, Copenhagen.

This editorial was published on September 1, 2019, at NEJM .org.
1. Kelbaek H, Terkelsen C], Helqvist S, et al. Randomized comparison of distal protection versus conventional treatment in primary percutaneous coronary intervention: the Drug Elution and Distal Protection in ST-Elevation Myocardial Infarction (DEDICATION) trial. J Am Coll Cardiol 2008;51:899-905.

2. Rasoul S, Ottervanger JP, de Boer MJ, et al. Predictors of 30day and 1-year mortality after primary percutaneous coronary intervention for ST-elevation myocardial infarction. Coron Artery Dis 2009;20:415-21.

3. Neumann F-J, Sousa-Uva M, Ahlsson A, et al. 2018 ESC/ EACTS Guidelines on myocardial revascularization. Eur Heart ] 2019;40:87-165.

4. Levine GN, Bates ER, Blankenship JC, et al. 2015 ACC/AHA/ SCAI focused update on primary percutaneous coronary intervention for patients with ST-elevation myocardial infarction: an update of the 2011 ACCF/AHA/SCAI guideline for percutaneous coronary intervention and the 2013 ACCF/AHA guideline for the management of ST-elevation myocardial infarction: a report of the American College of Cardiology/American Heart Association Task Force on Clinical Practice Guidelines and the Society for Cardiovascular Angiography and Interventions. Circulation 2016; 133:1135-47.

5. Wald DS, Morris JK, Wald NJ, et al. Randomized trial of preventive angioplasty in myocardial infarction. N Engl J Med 2013;369:1115-23.

6. Gershlick AH, Khan JN, Kelly DJ, et al. Randomized trial of complete versus lesion-only revascularization in patients undergoing primary percutaneous coronary intervention for STEMI and multivessel disease: the CVLPRIT trial. J Am Coll Cardiol 2015;65:963-72.

7. Engstrøm T, Kelbæk H, Helqvist $\mathrm{S}$, et al. Complete revascularisation versus treatment of the culprit lesion only in patients with ST-segment elevation myocardial infarction and multivessel disease (DANAMI-3-PRIMULTI): an open-label, randomised controlled trial. Lancet 2015;386:665-71.

8. Smits PC, Abdel-Wahab M, Neumann FJ, et al. Fractional flow reserve-guided multivessel angioplasty in myocardial infarction. N Engl J Med 2017;376:1234-44.

9. Mehta SR, Wood DA, Storey RF, et al. Complete revascularization with multivessel $\mathrm{PCl}$ for myocardial infarction. $\mathrm{N}$ Engl J Med 2019;381:1411-21.

10. Wallentin L, Becker RC, Budaj A, et al. Ticagrelor versus clopidogrel in patients with acute coronary syndromes. $\mathrm{N}$ Engl J Med 2009:361:1045-57.

DOI: 10.1056/ N EJMe1910898

Copyright @ 2019 Massachusetts Medical Sodiety.

\title{
A Metabolic Vulnerability of Vision
}

\author{
Henna Tyynismaa, Ph.D.
}

New therapies targeting metabolic vulnerabilities of specific tumor types have created wide interest in recent years. Through research now reported in the Journal by Gantner et al., ${ }^{1}$ metabolic precision therapy may become possible in patients with a rare eye disease, macular telangiectasia type 2 , which leads to a progressive loss of central vision in both eyes in middle-aged or older persons. ${ }^{2}$

The macula is the small area in the back of the eye that is responsible for high-resolution (i.e., sharp) vision. In the center of the macula is the fovea, which has the highest density of cone photoreceptor cells and thus, as compared with 
other parts of the macula, provides the highest degree of visual resolution. In persons with macular telangiectasia type 2, foveal photoreceptors degenerate, preventing the transduction of light signals to the brain. Telangiectasia refers to small dilated blood vessels, which are secondary abnormalities in this primarily neurodegenerative condition. There is currently no effective treatment for the condition, although encouraging results from a phase 2 trial of a neuroprotective agent, ciliary neurotrophic factor, were reported earlier this year, ${ }^{3}$ and an anti-vascular endothelial growth factor drug, bevacizumab, has been found to ameliorate secondary bloodvessel formation. ${ }^{4}$

A genetic component of macular telangiectasia type 2 was suspected, but the combination of genetic heterogeneity and environmental factors affecting the penetrance of genetic effect has made it challenging to establish clear inheritance patterns and to identify disease genes. A genomewide association study in combination with the analysis of blood metabolites from patients with macular telangiectasia type 2 provided some evidence to support involvement of the serine biosynthesis pathway in conferring susceptibility to the disease. ${ }^{5}$ Gantner et al. now report on specific genetic causes of macular telangiectasia type 2: variants in the genes SPTLCl and SPTLC, the protein products of which participate in sphingolipid metabolism. (Sphingolipids are a large family of lipids that among other roles, mediate a variety of metabolic, growth, and cellsurvival processes.)

It was previously established that pathogenic variation in SPTLC and SPTLC causes another neurodegenerative disease, hereditary sensory and autonomic neuropathy type 1 (HSAN 1), which affects the peripheral nerves and causes sensory abnormalities such as blunted nociception and variable limb weakness. ${ }^{6,7}$ Both genes are needed to make the key enzyme in sphingolipid synthesis, serine palmitoyltransferase (SPT). The etiologic mutations in SPTLCl and SPTLC do not reduce the activity of SPT but instead alter its substrate specificity from serine to alanine. This alteration results in the production and accumulation of deoxysphingolipids, ${ }^{8}$ which are neurotoxic.

Having obtained earlier evidence of possible disruption of serine metabolism in persons with macular telangiectasia type 2, with SPTLCl and SPTLC as strong candidate genes, the authors carefully examined 11 patients with HSAN 1 for macular telangiectasia type 2-associated macular changes and found such changes in 9 of the patients. Further studies in larger patient cohorts are needed to establish how commonly macular telangiectasia type 2 and sensory neuropathy co-occur. Is macular degeneration typical of all or only specific mutation types? Do any patients with macular telangiectasia type 2 who do not have SPTLCl or SPTLC mutations have neuropathic symptoms? What other monogenic causes (if any) in the same pathway might contribute to macular telangiectasia type 2? Why sensory neurons and foveal photoreceptors are especially vulnerable to elevated levels of deoxysphingolipids is another unanswered question, although a recent study provides a potential clue: the accrual of a specific type of deoxysphingolipid in the mitochondria promotes mitochondrial fission and glucose intolerance. ${ }^{9}$ This, in turn, may preferentially compromise the function of cells with high energy requirements, such as neurons (the photoreceptor is a type of neuron).

Gantner et al. went on to analyze 125 persons with macular telangiectasia type 2 who did not test positive for mutations affecting SPT. (As an aside, it seems from the comparatively large size of this group of patients that such mutations cause macular telangiectasia type 2 in a minority of affected persons). However, even in the absence of an SPTLC mutation, aberrant serine metabolism nonetheless appears to have a role in the pathogenesis of disease, because low serine and high deoxysphingolipid levels were found in the blood of the 125 patients with macular telangiectasia type 2 who did not have SPTLC or SPTLC mutations. The authors suggest that the low serine levels increase deoxysphingolipid levels by reducing substrate availability for SPT as a general mechanism. Testing of a serine-depleted diet in mice supported these findings: elevated levels of deoxysphingolipids developed in the mice, along with retinal and peripheral sensory deficits. Using human retinal organoids, the authors showed that deoxydihydroceramides, derivatives of deoxysphingolipids, are toxic to photoreceptors. Inhibition of ceramide synthesis or treatment with fenofibrate to 
stimulate deoxysphingolipid degradation prevented death of the photoreceptor cells.

Serine supplementation has been shown to reduce levels of deoxysphingolipids in humans. ${ }^{10,11}$ The results of a randomized, placebo-controlled trial of high-dose serine (400 mg per kilogram of body weight per day) to treat persons with HSAN 1 were, without sensitive biomarkers of disease progression, difficult to interpret. ${ }^{12}$ The serine-sphingolipid pathway now represents a target for experimental strategies in the treatment of macular telangiectasia type 2 , but as the authors rightly caution, it would be premature to prescribe serine or fenofibrate without a better understanding of disease etiology within the broader patient population. In the meantime, patients with HSAN1 should be evaluated for signs of macular telangiectasia type 2 , and vice versa.

Disclosure forms provided by the author are available with the full text of this editorial at NEJM.org.

From the Stem Cells and Metabolism Research Program, Faculty of Medicine, University of Helsinki, Helsinki, Finland.

This editorial was published on September 11, 2019, at NEJM .org.

1. Gantner ML, Eade K, Wallace M, et al. Serine and lipid metabolism in macular disease and peripheral neuropathy. N Engl J Med 2019;381:1422-33.

2. Charbel Issa P, Gillies MC, Chew EY, et al. Macular telangiectasia type 2. Prog Retin Eye Res 2013;34:49-77.
3. Chew EY, Clemons TE, Jaffe GJ, et al. Effect of ciliary neurotrophic factor on retinal neurodegeneration in patients with macular telangiectasia type 2: a randomized clinical trial. Ophthalmology 2019;126:540-9.

4. Roller AB, Folk JC, Patel NM, et al. Intravitreal bevacizumab for treatment of proliferative and nonproliferative type 2 idiopathic macular telangiectasia. Retina 2011;31:1848-55.

5. Scerri TS, Quaglieri A, Cai $C$, et al. Genome-wide analyses identify common variants associated with macular telangiectasia type 2. Nat Genet 2017;49:559-67.

6. Dawkins JL, Hulme DJ, Brahmbhatt SB, Auer-Grumbach M, Nicholson GA. Mutations in SPTLC1, encoding serine palmitoyltransferase, long chain base subunit-1, cause hereditary sensory neuropathy type I. Nat Genet 2001;27:309-12.

7. Rotthier $A$, Auer-Grumbach $M$, Janssens $K$, et al. Mutations in the SPTLC2 subunit of serine palmitoyltransferase cause hereditary sensory and autonomic neuropathy type I. Am J Hum Genet 2010;87:513-22.

8. Penno A, Reilly MM, Houlden $\mathrm{H}$, et al. Hereditary sensory neuropathy type 1 is caused by the accumulation of two neurotoxic sphingolipids. J Biol Chem 2010;285:11178-87.

9. Chaurasia B, Tippetts TS, Mayoral Monibas R, et al. Targeting a ceramide double bond improves insulin resistance and hepatic steatosis. Science 2019;365:386-92.

10. Auranen M, Toppila J, Suriyanarayanan S, et al. Clinical and metabolic consequences of L-serine supplementation in hereditary sensory and autonomic neuropathy type 1C. Cold Spring Harb Mol Case Stud 2017;3(6):a002212.

11. Garofalo K, Penno A, Schmidt BP, et al. Oral L-serine supplementation reduces production of neurotoxic deoxysphingolipids in mice and humans with hereditary sensory autonomic neuropathy type 1. J Clin Invest 2011;121:4735-45.

12. Fridman V, Suriyanarayanan $S$, Novak $P$, et al. Randomized trial of I-serine in patients with hereditary sensory and autonomic neuropathy type 1. Neurology 2019;92(4):e359-e370.

DO I: 10.1056/ N EJMe1910322

Copyright (c) 2019 Massadhusetts Medical Society. 